\title{
Small molecules against B-RAF (BRAF) Val600Glu (V600E) single mutation
}

This article was published in the following Dove Press journal:

International Journal of Nanomedicine

31 July 2015

Number of times this article has been viewed

\author{
Florin Zaharie ${ }^{1, *}$ \\ Roxana Cojocneanu- \\ Petric ${ }^{1, *}$ \\ Mihai Muresan' \\ loana Frinc ${ }^{2}$ \\ Delia Dima ${ }^{2}$ \\ Bobe Petrushev ${ }^{3}$ \\ Alina Tanase ${ }^{4}$ \\ Cristian Berce' \\ Mariana Chitic ${ }^{2}$ \\ loana Berindan-Neagoe ${ }^{\prime}$ \\ Valentina Pileczki' \\ Alexandru Irimie ${ }^{5}$ \\ Ciprian Tomuleasa ${ }^{2}$ \\ 'Iuliu Hatieganu University of \\ Medicine and Pharmacy, ${ }^{2}$ Department \\ of Hematology, Ion Chiricuta \\ Oncology Institute, ${ }^{3}$ Department \\ of Pathology, Emergency University \\ Hospital, Cluj Napoca, ${ }^{4}$ Department \\ of Stem Cell Transplantation, \\ Fundeni Clinical Institute, Bucharest, \\ ${ }^{5}$ Department of Surgery, Ion Chiricuta \\ Oncology Institute, Cluj Napoca, \\ Romania \\ *These authors contributed equally \\ to this communication
}

Correspondence: Ciprian Tomuleasa Department of Hematology, Ion Chiricuta Oncology Institute, Strada Mircea Eliade Nr 74, 400 I24, Cluj Napoca, Romania

Email ciprian.tomuleasa@umfcluj.ro

\section{Dear editor}

We have read with great interest the paper by Tang and $\mathrm{Chen}^{1}$ published in the most recent issue of the International Journal of Nanomedicine, in which the authors describe the protocol by which scientists constructed the ideal BRAF (V600E)-modeled structure through homology modeling and introduced the method of structure-based docking or virtual screening from a large compound database. They concluded that BRAF (V600E) has a quite prominent structural or conformational variation when compared to the wild-type BRAF protein by matrix of root mean square fluctuation and principal component analysis. On the basis of structure-based virtual screening, ligand-based quantitative structure activity relationship models, and molecular dynamics simulation, we recommend aknadicine and 16beta-hydroxy-19s-vindolinine $\mathrm{N}$-oxide as potent compounds for developing novel inhibitors in the future.

The v-raf murine sarcoma viral oncogene homolog B1 (BRAF) gene is mutated in $40 \%-60 \%$ of melanomas, the most common being the V600E mutation, which leads to activation of the mitogen activated protein kinase (MAPK) pathway. ${ }^{2} \mathrm{BRAF}$ is a member of the RAF family of serine/threonine protein kinases. This family consists of three kinases, ie, ARAF, CRAF (RAF-1), and BRAF, of which the latter has the highest basal kinase. BRAF functions to regulate the MAPK/ERK pathway, which is conserved in all eukaryotes. The RAS/RAF/MEK/ERK pathway acts as a signal transducer between the extracellular environment and the nucleus. Extracellular signals such as hormones, cytokines, and various growth factors interact with their receptors to activate the small G-proteins of the RAS family. BRAF-mutated tumors have a poor response to traditional chemotherapy and a poor prognosis in melanoma, thyroid, and colon cancers. ${ }^{3-5}$ Targeted therapies are of great interest for these types of cancer, and elucidation of the structure and functions of BRAF kinase is the subject of much ongoing research. The approach of targeting oncogenic kinases has been successful in the treatment of cancers with activating mutations in the kinase gene that drives their progression. It is likely that our evolving understanding of BRAF genetics and signaling will allow further personalization of cancer therapy with the goal of improving clinical responses.

We are confident that the results reported Tang and Chen ${ }^{1}$ have brought progress to the field, but targeting a malignant cell with a monotherapy protocol is expected to fail and thus lead to clinical relapse of the disease. Thus, combination therapy is likely to be the most effective management plan for the treatment of BRAF-mutated tumors. Many BRAF-specific inhibitors display a cytostatic response inducing senescence and are susceptible to acquired resistance. Therefore, combination with traditional chemotherapeutic agents seems to be more effective than either treatment alone. 


\section{Acknowledgments}

This communication is published under the framework of the European Social Fund, Human Resources Development Operational Programme 2007-2013 (project number POSDRU 159/1.5/138776) and is supported by internal grants from the Iuliu Hatieganu University of Medicine and Pharmacy awarded to FZ and RC-P.

\section{Author contributions}

All authors have made substantial contributions to conception and design, acquisition of data, or analysis and interpretation of data; took part in either drafting the communication or revising it critically for important intellectual content; gave final approval of the version to be published; and agree to be accountable for all aspects of the work in ensuring that questions related to the accuracy or integrity of any part of the work are appropriately investigated and resolved.

\section{Disclosure}

The authors report no conflicts of interest in this work.

\section{References}

1. Tang HC, Chen YC. Insight into molecular dynamics simulation of BRAF(V600E) and potent novel inhibitors for malignant melanoma. Int J Nanomedicine. 2015;10:3131-3146.

2. Penman CL, Faulkner C, Lowis SP, Kurian KM. Current understanding of BRAF alterations in diagnosis, prognosis, and therapeutic targeting in pediatric low-grade gliomas. Front Oncol. 2015;5:54.

3. Wheler J, Yelensky R, Falchook G, et al. Next generation sequencing of exceptional responders with BRAF-mutant melanoma: implications for sensitivity and resistance. BMC Cancer. 2015;15:61.

4. Pettirossi V, Santi A, Imperi E, et al. BRAF inhibitors reverse the unique molecular signature and phenotype of hairy cell leukemia and exert potent antileukemic activity. Blood. 2015;125:1207-1216.

5. Tiacci E, Schiavoni G, Martelli MP, et al. Constant activation of the RAF-MEK-ERK pathway as a diagnostic and therapeutic target in hairy cell leukemia. Haematologica. 2013;98:635-639. 


\section{Authors' reply \\ Hsin-Chieh Tang' \\ Yu-Chian Chen ${ }^{1-3}$}

'Department of Biomedical Informatics, Asia University, ${ }^{2}$ Human Genetic Center, Department of Medical Research, ${ }^{3}$ Research Center for Chinese Medicine and Acupuncture, China Medical University Hospital, Taichung, Taiwan

Correspondence: Yu-Chian Chen

Research Center for Chinese Medicine and Acupuncture, China Medical University Hospital, 2 Yude Rd, North District, Taichung City 404,

Taiwan

Tel +88 6422052121 ext 4306

Email ycc929@mit.edu

\section{Dear editor}

The RAS/RAF/MEK/ERK pathway is the most significant signal cascade when discussing the etiology of malignant melanoma. BRAF(V600E) is the most destructive single mutation which may lead to advanced or metastatic melanoma. Knowing the molecular characteristics and structural variation of $\mathrm{BRAF}(\mathrm{V} 600 \mathrm{E})$ protein when compared to wild-type BRAF protein by molecular dynamics simulation helps us to understand how the mutant protein amplifies the phosphorylation and metamorphic character.

There are many mechanisms of resistance with regard to existing BRAF inhibitors. RAS or MEK mutation is one of the acquired responses conferring drug resistance. Gln61 (Q61) mutation of N-ras or Cys121 (C121) mutation of MEK is an example of drug resistance. Our previous study entitled "Molecular insight and resolution for tumors harboring the H-ras $(\mathrm{G} 12 \mathrm{~V})$ mutation" investigated the H-ras protein single mutation and reported that $\mathrm{H}-\mathrm{ras}(\mathrm{G} 12 \mathrm{~V})$ mutation offered a stable condition for excess signal transduction. Our subsequent studies have focused on N-ras and MEK single mutation. Through structure-based analysis, ligand-based analysis, or molecular dynamics simulation, we can explore the differences between these wild-type and mutant proteins, and provide possible resolution for tumors harboring N-ras or MEK mutation. ${ }^{2}$

In addition to acquired resistance, a compensatory increase in activity of microphthalmia-associated transcription factor is involved in adaptive resistance. Further, BRAF mutations are not detected in approximately $50 \%$ of melanoma lesions. Cyclin-dependent kinase 2 is the alternative target for drug management of these melanoma patients. Our previous study entitled "Drug design of cyclin-dependent kinase 2 inhibitor for melanoma from traditional Chinese medicine" elaborated another possible therapy for advanced or metastatic melanoma. ${ }^{3}$

\section{Disclosure}

The authors report no conflicts of interest in this work.

\section{References}

1. Hartsough E, Shao Y, Aplin AE. Resistance to RAF inhibitors revisited. J Invest Dermatol. 2014;134(2):319-325.

2. Tang HC, Chen YC. Molecular insight and resolution for tumors harboring the H-ras (G12V) mutation. RSC Adv. 2015;5(27):20623-20633.

3. Tang HC, Chen CY. Drug design of cyclin-dependent kinase 2 inhibitor for melanoma from traditional Chinese medicine. Biomed Res Int 2014;2014:798742.

Dove Medical Press encourages responsible, free and frank academic debate. The content of the International Journal of Nanomedicine 'letters to the editor' section does not necessarily represent the views of Dove Medical Press, its officers, agents, employees, related entities or the International Journal of Nanomedicine editors. While all reasonable steps have been taken to confirm the content of each letter, Dove Medical Press accepts no liability in respect of the content of any letter, nor is it responsible for the content and accuracy of any letter to the editor.

\section{Publish your work in this journal}

The International Journal of Nanomedicine is an international, peerreviewed journal focusing on the application of nanotechnology in diagnostics, therapeutics, and drug delivery systems throughout the biomedical field. This journal is indexed on PubMed Central, MedLine, CAS, SciSearch $\AA$, Current Contents ${ }^{\circledR} /$ Clinical Medicine,
Journal Citation Reports/Science Edition, EMBase, Scopus and the Elsevier Bibliographic databases. The manuscript management system is completely online and includes a very quick and fair peer-review system, which is all easy to use. Visit http://www.dovepress.com/ testimonials.php to read real quotes from published authors. 